\title{
Ocorrência e perfil de suscetibilidade de Candida sp em hemoculturas de um hospital universitário
}

\section{Occurrence and susceptibility profile of Candida sp in blood cultures from a university hospital}

Ana Beatriz A. Teixeira-Loyola ${ }^{1 *}$; Ana Paula Fernandes²; Camila C. Barbosa²; João Marcelo A. Mattozo ${ }^{3}$; Adenilza C. Silva ${ }^{4}$ Angélica Z. Schreiber

\begin{abstract}
RESUMO
O gênero Candida é responsável por infecções associadas à fungemias hospitalares, principalmente em setores críticos. Objetivos: avaliar a ocorrência de Candida $s p$ de hemoculturas do Laboratório de Análises Clínicas, do HCSL de Pouso Alegre, M G e avaliar o perfil de suscetibilidade frente a anfotericina B, itraconazol, fluconazol e voriconazol. Metodologia: Foi realizado um estudo descritivo de julho de 2009 á julho de 2010, onde foram avaliadas todas as hemoculturas do HCSL deste período. Os fungos isolados das hemoculturas foram identificados por metodologia clássica e automatizada. O teste de suscetibilidade foi realizado pela técnica de microdiluição em caldo segundo o documento CLSI M27A3. Resultados: Foram avaliadas 1388 amostras sendo 108 amostras positivas, e destas, 10 fúngicas. A ocorrência fúngica foi: UTI adulto (4), UTI Neonatal (5) e Enfermaria Masculina (1). Foram identificadas Candida albicans (5) e Candida parapsilosis (4), e Candida sp (1). Todos os micro-organismos apresentaram sensibilidade frente aos antifúngicos testados. Conclusão: O percentual de positividade para candidemia observada neste estudo $(1,38 \%)$ é similar aos estudos comparativos como pudemos observar na incidência em unidades de terapia intensiva. Vale destacar que a ocorrência é superior à observada em trabalhos internacionais.
\end{abstract}

Palavras-chave: Candidemia. Fungemia. Diagnóstico. Sangue.

\section{Introdução}

O gênero Candida apresenta-se como principal responsável por infecções associadas à fungemias hospitalares, principalmente em setores críticos, como as unidades de terapia intensiva (UTIs). ${ }^{1}$
A expansão do número de pacientes críticos tem determinado o aparecimento de novas espécies de Candida. ${ }^{2}$

Entre as infecções invasivas causadas pelo gênero Candida, vale salientar a relevância clínica dos casos de infecção de corrente sanguínea, complica-
1. Doutorado. Universidade do Vale do Sapucaí (Univás); Pouso Alegre-MG.

2. Discente do Curso de Farmácia, aluna de iniciação científica$\mathrm{PIBIC/Univás}$

3. Farmacêutico- Bioquímico. Laboratório de Análises Clínicas do Hospital das Clínicas Samuel Libânio, Pouso Alegre-MG

4. Farmacêutica, aluna de mestrado em Ciências Médicas da Unicamp.

5. Doutorado. Unicamp - Campinas-SP
${ }^{*}$ Correspondência com o autor: Universidade do Vale do Sapucaí - UnivásUnidade Central. Avenida Coronel Alfredo Custódio de Paula, 320 CEP 37550-000, Pouso Alegre, Minas Gerais Fone: (35) 3449-9271, (35) 3449-8772 E-mail: analkmim@ hotmail.com, analkmim@gmail.com

Artigo recebido em 08/04/2013 Aprovado para publicação em 16/10/2013 
ção esta conhecida como candidemia ou candidíase hematogênica. Na verdade, o termo candidíase hematogênica engloba um espectro amplo de situações clínicas, incluindo desde episódios isolados de candidemia até casos onde o fungo presente na corrente sanguínea dissemina-se para um ou vários órgãos do hospedeiro infectado. ${ }^{3}$

Acredita-se que a maioria dos casos de candidemia seja adquirida por via endógena, pela translocação do patógeno através do trato gastrointestinal, local onde há rica colonização por Candida spp em até $70 \%$ da população normal. Sendo assim, fatores que aumentem a colonização intestinal por Candida (uso de antibióticos, íleo, oclusão intestinal) ou determinem atrofia ou lesão de mucosa intestinal (jejum prolongado, nutrição parenteral total, hipotensão, quimioterapia) podem potencializar o fenômeno de translocação no tubo gastrointestinal. ${ }^{3}$

Infecções hematogênicas por Candida spp também podem ser adquiridas por via exógena, através do contato das mãos de profissionais de saúde, com pacientes portadores de catéteres vasculares em posição central, implante de próteses contaminadas, bem como pela administração parenteral de soluções contaminadas. ${ }^{1}$

A capacidade dos fungos de causar doenças invasivas é muito bem documentada: as espécies de Candida constituem, hoje, o quarto micro-organismo mais isolado nas hemoculturas de pacientes internados nos Estado Unidos, ${ }^{4}$ sendo este responsável por cerca de $80 \%$ das infecções fúngicas no ambiente hospitalar, constituindo causa relevante das infecções de corrente sanguínea, respondendo por cerca de $8 \%$ dos casos das infecções documentadas neste sítio. ${ }^{3}$

A hemocultura representa um importante método laboratorial de diagnóstico das fungemias. ${ }^{5}$

Porém, o diagnóstico das espécies de Candida spp pode ser problemático por ausência de sintomas clínicos específicos e demora em obter resultado pelos métodos diagnósticos tradicionais. Esse quadro facilita a disseminação do fungo por múltiplos órgãos, resultando em piora clínica do paciente, ampliação dos efeitos adversos, administração medicamentosa prolongada e, consequentemente, aumento dos custos hospitalares. ${ }^{6}$

O reconhecimento precoce das fungemias por Candida é cada vez mais necessário, uma vez que o paciente envolvido geralmente apresenta o sistema imunológico comprometido. ${ }^{5}$
Infecções invasivas causadas por essas leveduras associam-se a internação prolongada (três a 30 dias), alta taxa de mortalidade (10\% a $49 \%$ ) e elevado custo hospitalar. Em casos de infecção grave e sistêmica, a melhora ou até a sobrevivência do paciente depende da rápida identificação do patógeno e, consequentemente, da introdução precoce da terapia antifúngica. $^{3}$

Em infecções fúngicas provocadas por Candida sp, a identificação de sua espécie é essencial, uma vez que a patogenicidade e o perfil de sensibilidade a um determinado antifúngico são variáveis entre as diferentes espécies. Apesar de a Candida albicans ser a espécie mais comumente isolada nas infecções superficiais ou invasivas, a incidência de infecções provocadas por Candida não-albicans é crescente. $^{6}$

O laboratório de microbiologia hospitalar é a primeira linha de defesa frente à disseminação de agentes nosocomiais. Além da correta identificação microbiana, é papel dos laboratórios detectarem a resistência aos antimicrobianos de uso clínico. ${ }^{7}$

A ocorrência de casos de candidemia em hospitais terciários aumentou substancialmente nas últimas décadas em diferentes partes do mundo. ${ }^{6}$

No período de 1986 a 1990, já se encontrava entre os cinco primeiros agentes mais comumente identificados em hemoculturas de pacientes internados em hospitais americanos.

Outro aspecto relevante a ser considerado, em relação aos episódios de candidemia, é o custo decorrente do atendimento ao paciente. Estudo realizado nos Estados Unidos da América definiu como sendo de US\$44.536 o custo relacionado ao atendimento privado de cada paciente com candidemia, sendo que a maior parte dos gastos está relacionada ao maior tempo de internação necessário para atendimento destes pacientes. ${ }^{3}$

No Brasil, os dados sobre infecção fúngica hospitalar são escassos e pouco abrangentes. Além disto, esses dados não são consolidados pela maioria dos hospitais, dificultando ainda mais o conhecimento da dimensão do problema no país. ${ }^{3}$

O Hospital das Clínicas Samuel Libânio (HCSL) é um hospital terciário e está situado na cidade de Pouso Alegre, no estado de Minas Gerais, região sudeste do Brasil, com capacidade para 300 leitos.

Este panorama justifica a importância do conhecimento, por parte dos profissionais de saúde, das 
medidas necessárias para o diagnóstico, controle e tratamento de infecções invasivas por Candida spp. ${ }^{3}$

O objetivo deste trabalho foi avaliar a ocorrência de espécies de Candida sp. provenientes de hemoculturas do Laboratório de Análises Clínicas, setor Microbiologia do Hospital das Clínicas Samuel Libânio de Pouso Alegre, Minas Gerais; identificar as espécies de Candida isoladas das hemoculturas pela técnica clássica e método automatizado, avaliar a suscetibilidade dos isolados frente aos antifúngicos anfotericina $\mathrm{B}$, itraconazol, fluconazol e voriconazol e avaliar os prontuários de pacientes hospitalizados para a obtenção de dados referentes ao gênero, idade do paciente, local de internação e mês e ano do procedimento laboratorial submetidos no Laboratório de Análises Clínicas do Hospital das Clínicas Samuel Libânio.

\section{Material e métodos}

Foi realizado um estudo transversal, observacional, descritivo e individual, num período de um ano (julho de 2009 a julho de 2010). Foram avaliadas todas as hemoculturas referentes aos pacientes do Hospital das Clínicas Samuel Libânio incluindo a maioria das especialidades da clínica médica e cirúrgica, unidade de terapia intensiva (UTI) de adulto (clínica e cirúrgica), UTI neonatal, UTI pediátrica, serviço de pronto atendimento e emergência de adulto e pediátrico, ginecologia e obstetrícia. Das hemoculturas positivas para Candida sp foram avaliados os prontuários dos pacientes hospitalizados. Dos prontuários foram obtidos dados como gênero e idade do paciente, local de internação, juntamente com mês e ano do procedimento laboratorial.

Isolamento: No Laboratório de Análises Clínicas, setor Microbiologia do Hospital das Clínicas Samuel Libânio utiliza-se para hemoculturas o sistema Hemocult ${ }^{\circledR}$, PROBAC composto por um laminocultivo com duas faces acopladas á parte superior de um recipiente plástico contendo um caldo suplementado com extrato de levedura e polianetol-sulfunato de sódio (SPS) e acrescido de substâncias orgânicas e inorgânicas para neutralização de antimicrobianos. As faces do laminocultivo são: ágar chocolate e próximo à tampa o indicador de gás carbônico que detecta o crescimento de bactérias e fungos. Já na face dividida encontra-se ágar Sabouraud e ágar MacConkey. O laminocultivo vem desconectado da parte inferior (caldo), podendo ser realizada esta conexão no momento da chegada do frasco ao laborató- rio independente do tempo decorrido da coleta. A conexão deve ser realizada antes da incubação do sistema. ${ }^{8}$

O Laminocultivo Hemocult ${ }^{\circledR}$, PROBAC foi observado duas vezes ao dia quanto o aparecimento de colônias e/ou mudança do indicador.

O tempo de incubação máxima da hemocultura foi de seis dias, sendo considerada negativa para bactéria após este período.

Para pesquisa de fungos, foi incubada por mais dez a doze dias totalizando vinte dias, em temperatura ambiente.

Para as hemoculturas positivas, foi realizada técnica de Gram e cultivo em Agar Sabouraud dextrose.

Identificação: Os fungos isolados foram identificados de acordo com metodologia clássica. ${ }^{9}$

As amostras também foram enviadas á Unicamp para identificação automatizada pelo Vitek $2 ®$, BIOMÉRIEUX que emprega cartões para a identificação de leveduras.

Perfil de suscetibilidade: $\mathrm{O}$ teste de microdiluição em caldo foi realizado conforme o documento M27-A3. ${ }^{10}$ Os antifúngicos testados frente às Candidas sp identificadas foram anfotericina B, itraconazol, fluconazol e voriconazol nas diferentes concentrações que variaram de $0,125 \mu \mathrm{g} / \mathrm{mL}$ a 128,0 $\mu \mathrm{g} / \mathrm{mL}$. Foram utilizadas as cepas padrões Candida krusei ATCC 6258 e Candida parapsilosis ATCC 22019 para validação dos testes.

\section{Resultados}

As hemoculturas enviadas ao setor de microbiologia do Laboratório de Análises Clínicas do HCSL recebem amostras em duplicatas de pacientes adultos e amostras únicas de pacientes pediátricos. Durante o período de execução deste trabalho foram avaliadas um total de 1388 hemoculturas das quais 1290 (93\%) foram negativas e $108(7,78 \%)$ amostras obtiveram crescimento, sendo que 98 (7\%) foram positivas para crescimento bacteriano ${ }^{11}$ e $10(1,38 \%)$ amostras positivas para fungos .

Das $108(100 \%)$ amostras que obtiveram crescimento, $46(40 \%)$ amostras foram isoladas de pacientes do gênero feminino, sendo 4 fúngicas $(3,7 \%)$ e $62(60 \%)$ amostras do gênero masculino, sendo 6 fúngicas $(5,5 \%)$.

A ocorrência fúngica por Candida spp foi registrada na Unidade de Terapia Intensiva Adulto (4), 
Unidade de Terapia Intensiva Neonatal (5) e Enfermaria Masculina (1).

Das $10(9,2 \%)$ amostras positivas para fungos, $5(4,6 \%)$ amostras foram identificadas como sendo de Candida albicans, $4(3,7 \%)$ de Candida parapsilosis e $1(0,9 \%)$ de Candida $s p$, de acordo com a identificação automatizada.

Os micro-organismos isolados mostraram sensibilidade in vitro frente aos antifúngicos testados (Tabela 1).

\section{Discussão}

No Brasil, Colombo e cols conduziram um estudo epidemiológico reunindo dados sobre infecções de corrente sanguínea documentadas em quatro hospitais da cidade de São Paulo, Candida spp respondeu por $4,3 \%$ do total das infecções de corrente sanguínea. $^{3}$

Em nosso estudo detectamos a ocorrência de $10(1,38 \%)$ hemoculturas positivas para Candida, sendo que $50 \%$ são da espécie albicans e $40 \%$ de não-albicans (C. parapsilosis). Colombo et al (2007), ${ }^{12}$ apontaram na detecção de 145 candidemias em 6 hospitais brasileiros o predomínio de espécies não-albicans em $63 \%$ dos casos, sendo Candida parapsilosis e Candida tropicalis as preponderantes, embora nas três últimas décadas em hospitais universitários americanos, Candida albicans tenha sido a espécie de Candida responsável pelo aumento de seis vezes mais na frequência de fungemias por esta levedura. ${ }^{12}$

No Brasil, C. albicans tem sido igualmente a levedura mais isolada de candidemias em todas as regiões do país. ${ }^{13}$ A explicação para o fato de $C$. albicans apresentar os maiores percentuais de recuperação pode estar relacionada à sua grande adaptabilidade e versatilidade patogênica. ${ }^{14}$

Ainda em nosso estudo, conduzido em Pouso Alegre, sul de Minas Gerais, foi possível observar que o número de amostras de hemocultura que o Laboratório de Microbiologia recebe, é homogêneo, sofrendo pouca variação no decorrer da pesquisa.

Percebe-se também que, quanto à preponderância, o gênero masculino é maioridade. Em geral, em estudos comparativos, a prevalência tem sido do gênero masculino, inclusive em pacientes pediátricos. ${ }^{15}$ BASSETTI et al, também confirmam a presente casuística, apesar de não haverem diferenças clínicas consideráveis para estes gêneros. ${ }^{16}$

Outro fato que deve ser levado em consideração é o aparecimento de novas espécies de Candida, estas que, uma vez oportunistas, são praticamente impossíveis de serem detectadas no começo da manifestação. Para tanto, os hospitais devem estar preparados para identificar correta e rapidamente toda e qualquer forma de Candida presente.

De acordo com Hazen, candidíases invasivas em humanos são causadas por cerca de 17 espécies. ${ }^{17}$ Entretanto, mais de 90\% destas infecções são atribu-

\section{Tabela 1}

Teste de suscetibilidade de Candida spp frente a anfotericina B, itraconazol, fluconazol e voriconazol.

\begin{tabular}{clcccc}
\hline Isolado & Identificação & $\begin{array}{c}\text { Anfotericina B } \\
\text { IC100 }\end{array}$ & $\begin{array}{c}\text { Fluconazol } \\
\text { IC 50 }\end{array}$ & $\begin{array}{c}\text { Itraconazol } \\
\text { IC 50 }\end{array}$ & $\begin{array}{c}\text { Voriconazol } \\
\text { IC 50 }\end{array}$ \\
\hline $\mathbf{2}$ & Candida albicans & 0,5 & 0,25 & 0,125 & $\leq 0,0156$ \\
$\mathbf{3}$ & C. parapsilosis & 1 & 0,5 & 0,25 & $\leq 0,0156$ \\
$\mathbf{4}$ & C. parapsilosis & 1 & 1 & 0,125 & $\leq 0,0156$ \\
$\mathbf{5}$ & Candida albicans & 1 & 0,125 & 0,125 & $\leq 0,0156$ \\
$\mathbf{7}$ & Candida albicans & 1 & 0,125 & 0,125 & $\leq 0,0156$ \\
$\mathbf{8}$ & C. parapsilosis & 0,5 & 0,25 & 0,125 & $\leq 0,0156$ \\
$\mathbf{9}$ & Candida albicans & 1 & 0,25 & 0,125 & $<0,0156$ \\
$\mathbf{1 0}$ & C. parapsilosis & 1 & 0,5 & 0,125 & $\leq 0,0156$ \\
\hline
\end{tabular}


ídas, basicamente, há apenas 5 delas: $C$. albicans, $C$. glabrata, C. krusei, C. parapsilosis e C. tropicalis. ${ }^{2}$

Para a identificação no presente estudo, as cepas foram enviadas para a UNICAMP, já que o HCSL não possui automatização para fungos, realizando apenas prova do tubo germinativo, prova esta, que somente detecta candida albicans sugestivamente, não identificando as outras espécies.

A expansão do número de pacientes críticos tem determinado o aparecimento de novas espécies de Candida. $^{2}$

Este fato tem imposto aos laboratórios de microbiologia hospitalar a necessidade de melhorias em sua capacidade de identificação de espécies patogênicas, não somente para suporte à terapêutica das doenças infecciosas, mas também para seu controle e prevenção. $^{7}$

O sucesso dos programas de vigilância das infecções hospitalares depende da competência do laboratório de microbiologia. ${ }^{18}$

A habilidade de identificar acuradamente microorganismos hospitalares é fundamental para qualquer sistema de vigilância em saúde, pois as informações geradas nos laboratórios de microbiologia condicionam as ações de controle, bem como influenciam as políticas e diretrizes dos programas institucionais e governamentais. ${ }^{19}$

Com a emergência das candidíases invasivas em pacientes imunocomprometidos, tem havido um crescente interesse pelos antifúngicos, bem como pelos métodos laboratoriais de detecção da susceptibilidade que, indubitavelmente, auxiliam na escolha da melhor droga a ser empregada e/ou na análise de eventuais fracassos terapêuticos. ${ }^{20}$
Em resumo, o presente trabalho contribuiu para o conhecimento da ocorrência das espécies de Candida isoladas de hemoculturas no HCSL de Pouso Alegre-MG.

Estes 10 resultados positivos reforçam ainda a importância da realização de estudos de vigilância para a documentação do perfil de susceptibilidade aos antifúngicos testados.

\section{Conclusão}

O percentual de positividade para candidemia observada neste estudo (1,38\%) foi similar aos estudos de Colombo et al, Ribeiro et al e França et al, como observado em unidades de terapia intensiva. ${ }^{3,5,21}$

Embora não existam explicações bem definidas, acredita-se que a introdução de práticas simples de controle de infecção hospitalar, como a higienização das mãos, e possivelmente profilaxia antifúngica em pacientes de risco, o panorama possa ser modificado.

Diante deste patamar, sugere-se a implantação de um setor de Micologia para o HCSL, já que é um hospital que recebe pacientes de toda a região, tendo, portanto, perfil para suprir esta deficiência, garantindo ainda mais a saúde e o bem- estar da população, diminuindo custos em internação e/ou antibioticoterapia.

\section{Agradecimentos}

Ao Programa Institucional de Bolsas de Iniciação Científica- PIBIC Univás. A Luzia Lyra Fernandes (Unicamp) e Prof. Marcos Mesquita Filho (Univás) pelo suporte técnico.

\begin{abstract}
The genus Candida is responsible for hospital infections associated with fungemia, especially in critical sectors. Objectives: To evaluate the prevalence of Candida species from blood cultures of Clinical Laboratory, the HCSL Pouso Alegre, MG and evaluated the susceptibility test for anphotericin B, itraconazole, fluconazole e voriconazole. Methods: We conducted a study from July 2009 to July 2010, which evaluated all blood cultures of HCSL this period. The fungi isolated from blood cultures were identified by classical methods and for automated identification. The susceptibility test was evaluated by microdilution broth CLSI M27-A3. Results: A total of 1388 samples with 108 positive samples, and fungal 10. The fungal incidence was: Adults UTI (4), Neo UTI (5) and Male Ward (1). Were identified as Candida albicans (5) and Candida parapsilosis (4), and Candida sp (1). All samples show sensibility for antifungal tested. Conclusion: The candidemia observed in this study $(1.38 \%)$ is similar to comparative studies as we have seen in the incidence in intensive care units.
\end{abstract}

Key-words: Candidemia. Fungemia. Diagnosis. Blood. 


\section{Referências bibliográficas}

1. Hinrichsen SL, Falcão E, Vilella T.A.S, Colombo AL, Nucci M, Moura L. et al. Candidemia em um hospital terciário do nordeste do Brasil. Rev Soc Bras Med. Trop. 2008, 41: 394-8.

2. Pfaller MA, and Diekema DJ. Epidemiology of invasive candidiasis: a persistent public health problem. Clin Microbiol Rev. 2007, 20: 133-63.

3. Colombo AL, Guimarães T. Epidemiologia das Infecções Hematogênicas por Candida spp. Rev Soc Bras Med Trop. 2003, 36: 599-607.

4. Figueiredo GC, Figueiredo ECQ. Tavares-Neto J. Aspectos Clínicos e terapêuticos da Osteomielite Vertebral por Fungos- Análise Secundária de Dados. Rev Bras Reumatol. 2007, 47: 36-41.

5. Ribeiro EL, Castro KRC, Noleto MB, Ferreira WM, Cardoso CG, Naves PLF. Ocorrência de Leveduras de Candida em Hemoculturas originadas de Infecções Nosocomiais. NewsLab. 2003, 60: 78-83.

6. Mímica LMJ, Ueda SMY, Martino MDV, Navarini A, Martini IJ. Diagnóstico de infecção por Candida: avaliação de testes de identificação de espécies e caracterização do perfil de suscetibilidade. J Bras Patol Med Lab. 2009, 45: 50-5.

7. Pfaller MA, and Diekema DJ. Role of Sentinel Surveillance of Candidemia: trends in species distribution and antifungal Susceptibility. J Clin Micorbiol. 2002, 40: 3551-7.

8. Koneman EW, Allen SD. Janda WM, Schreckenberger PC, Winn WC. Color Atlas and Textbook of Diagnostic Microbiology, 6th. ed. JB Philadelphia: Linpcott Company Press, 2006.

9. Lacaz CS, Porto E, Martins JEC. Micologia Médica: Fungo, Actinomicetos e Algas de Interesse Médico. 1991; 3:31-58.

10. Clinical Laboratory Standard Institute. Reference Method for Broth Dilution Antifungal Susceptibility Testing of Yeasts; approved standard, Third edition CLSI document M27-A3, 2008. National Committee for Clinical Laboratory Standards, Wayne, $\mathrm{Pa}$

11. Fernandes AP, Silva CJ, Costa C, Schreiber AZ, Mello FA, Teixeira-Loyola ABA. Incidência Bacteriana em Hemoculturas no Hospital das Clínicas Samuel Libânio de Pouso Alegre MG.Revista Eletrônica Acervo Saúde. 2011, 2: 122-33
12. Colombo AL. Diagnóstico de doenças Fúngicas Oportunistas: O Grande Desafio para os Centros Médicos de Atendimento Terciário. Prat Hosp. 2007; 52: 50-5.

13. Passos XS, Costa CR, Araújo CR, Nascimento ES, e Souza LK, Fernandes Ode F, Sales WS, Silva Mdo R: Species distribution and antifungal susceptibility patterns of Candida spp. bloodstream isolates from a Brazilian tertiary care hospital. Mycopathologia. 2007, 163:145-51.

14. Kumamoto CA, Vinces MD. Contributions of hyphae and hypha-co-regulated genesto Candida albicans virulence. Cell Microbiol. 2005; 7:1546-54.

15. Velasco E, Bigni R. A prospective cohort study evaluating the prognostic impact of clinical characteristics and comorbid conditions of hospitalized adult and pediatric cancer patients with candidemia. Eur J Clin Microbiol Infect Dis. 2008; 27:10718.

16. Bassetti M, Righi E, Costa A, Fasce R, Molinari MP, Rosso et al. Epidemiological trends in nosocomial candidemia in intensive care. BMC Infect. Diseases. 2006; 10: 6-21.

17. Hazen K C. New and emerging yeast pathogens. Clin Microbiol Rev. 1995, 8: 462-78.

18. Peterson L R, Noskin G A. New technology for detecting multidrug-resistant pathogens in the clinical microbiology laboratory. Emerg Infect Dis. 2001; 7: 306-11.

19. Rosenthal VD, Maki DG, Mehta A, et al. International Nosocomial Infection Control Consortium report, data summary for 20022007, issued January 2008. Am J Infect Control. 2008; 36: 627-37.

20. Rodero L, Córdoba S, Vivot W, Campo M, Corfield, P, Olguín C., et al. Disk diffusion method for fluconazole susceptibility testing of Candida spp. isolates. Rev Argent Microbiol. 2006; 38: $155-63$.

21. França JCB, Ribeiro CEL, Queiroz-Telles F. Candidemia em um hospital terciário brasileiro: incidência, frequência das diferentes espécies, fatores de risco e suscetibilidade aos antifúngicos. Prat Hosp. 2008; 60: 23-8. 